\title{
Advances in the Study of Posture, Balance and the Brain
}

This is a collection of papers presented at the International Workshop on Posture, Balance and the Brain, held on $13^{\text {th }}$ September 2014 at the School of Physical Education and Sport Sciences, Aristotle University of Thessaloniki, Thessaloniki, Greece. The idea for this workshop emerged after the visit of Prof. Vassilia Hatzitaki from Motor Control and Learning Laboratory in Sofia on the invitation of our research team for the project "Cortical Regulation of the Quiet Stance during Sensory Conflict," supported by the National Science Fund (NSF), Ministry of Education, Youth and Science, Republic of Bulgaria, Grant TK 02/60. During this memorable visit we found common views and goals, setting a firm foundation for further fruitful collaboration. This workshop was also the final event of the NSF project led by me, and I wish to thank once again Vassilia and her colleagues for their generous hospitality, tireless support and friendship.

Standing balance plays a major supportive role in daily life, professional and sport activities. While most healthy adults take this ability for automatic and granted, stance maintenance is very complex and can be a major challenge for older persons, those living with pathologies or those moving across extreme environmental conditions. Over the past decade there has been remarkable progress in the field of standing, especially concerning cortical control of standing balance. The papers of Gatev et al. ${ }^{1}$ and Dimitrov et al. ${ }^{2}$ in this book show that during sensory-conflicted stance due to absence of vision and/or inadequate vestibular information both EEG sources and EEG mapping differ from those during quiet stance with all sensory modalities available and provide adequate information for the static standing balance. As Stambolieva and colleagues demonstrate, the specific training process of archers makes them less dependent on vision for their equilibrium, ${ }^{3}$ which suggests that static standing balance can be trained to fit to the archers' task and in this process the cortical regulation most probably plays a substantial role. In addition, Gatev and Kirilova show that sensory conflict influences the dynamic stance strategy during functional reach task, as well as the task achievement, ${ }^{4}$ while Minchev, Kelevedjiev and Gatev demonstrate that audio-visual stimulation leads to cortical entrainment, which may in turn change the pattern of stance equilibrium. ${ }^{5}$ Although new treatments, training and rehabilitation procedures are lagging behind our understanding, the growing sophistication of balance studies is starting to provide clinicians, rehabilitators and trainers with bona fide therapeutic targets. ${ }^{3,5,6}$ 
A comprehensive update and revision of standing balance control led to the development of models of static and dynamic standing balance. The paper of Sofianidis and Hatzitaki deals with the interpersonal synchrony in a dancing couple being in light touch contact. ${ }^{7}$ The authors found that experts in traditional dances show stronger spontaneous interpersonal synchrony when sway is paced by an auditory metronome and can better integrate light touch information with auditory time cues, and are less influenced by light touch interference. This suggests that expertise is a possible tool to solve sensory conflict.

Finally, utmost thanks go to the contributing authors for their timely contributions to this book. With increasing pressures upon academicians to generate clinical or education revenue and/or grant money, time for thoughtful review of the literature and creative synthesis in the form of exceptional, insightful, and motivating papers like the ones in the present book has become increasingly precious. As a head of the project, I tried to do my best to be patient and persistent from start to finish. I want to express deep gratitude to my PhD student Ms. Katerina Kirilova, who cheerfully and professionally helped with formatting and editing the book. Special thanks also go to all members of my project research team and Dr. Vassilia Hatzitaki and her colleagues from the Motor Control and Learning Laboratory, Department of Physical Education and Sports Science, Aristotelian University of Thessaloniki for their support, inspiration and collaboration.

I hope this book, which brings theoretical and practical approaches to the research on standing balance, will be an invaluable resource to scientists, basic researchers, clinicians, sport trainers and others interested in assuring the adequate base for postural and motor control to cope with task and environmental challenges during daily life and professional tasks. Additional information can be found on the websites: http://gaitandposture.web.auth.gr/cms/ and http://cleverstance.com.

Sofia,

\section{Plamen Gatev, MD, PhD}

On behalf of the School of Physical Education and Sport Sciences of Aristotle University, I would like to thank Prof. Gatev and his colleagues for their visit to Thessaloniki and the successful delivery of the "Posture, Balance and the Brain" workshop on Saturday, 13 September 2014. The workshop was one of the most successful and stimulating educational events of the past year for our school. Our staff, graduate and undergraduate students had the opportunity of learning about the cortical control of standing balance, the contribution of sensory systems in postural control and how this can be modulated by athletic experience. More importantly, this knowledge was delivered through the presentation of the latest research findings from fellows with a well-known history and very respectful tradition in the study of the neurophysiology of motor control. 
It was a very inspiring surprise to realize the sharing of common interests and how our research in posture and balance complements the long standing work performed by Prof. Gatev and his team in the Bulgarian National Academy of Sciences. The workshop provided the opportunity for very fruitful exchanges of ideas and transfer of knowledge and built the grounds for a productive future collaboration in the search for the optimal balance control.

Thessaloniki,

\section{Vassilia Hatzitaki, PhD, Associate Professor}

Motor Control and Learning Laboratory, Aristotle University of Thessaloniki

\section{References}

1. Gatev P, Stambolieva K, Lalova J, Dimitrov B. EEG Sources during Quiet and Sensory-Conflicted Stance. In: Gatev P, Hatzitaki V, editors. Posture, Balance and the Brain. Sofia: Procon; 2015. p. 7-14. doi: 10.11610/posture.02.

2. Dimitrov B, Stambolieva K, Gatev P. EEG Mapping during Quiet and Sensory-Conflicted Stance. In: Gatev P, Hatzitaki V, editors. Posture, Balance and the Brain. Sofia: Procon; 2015. p. 15-21. doi: 10.11610/posture.03.

3. Stambolieva K, Otzetov M, Petrova D, Ikonomov R, Gatev P. Postural Stability during Static Upright Stance in Archers. In: Gatev P, Hatzitaki V, editors. Posture, Balance and the Brain. Sofia: Procon; 2015. p. 29-35. doi: 10.11610/posture.05.

4. Gatev P, Kirilova K. Sensory Conflict Influence on Functional Reach Performance \& Dynamic Standing Balance. In: Gatev P, Hatzitaki V, editors. Posture, Balance and the Brain. Sofia: Procon; 2015. p. 22-28. doi: 10.11610/posture.04.

5. Minchev Z, Kelevedjiev E, Gatev P. Audio-Visual Entrainment Influence on Postural Dynamics. In: Gatev P, Hatzitaki V, editors. Posture, Balance and the Brain. Sofia: Procon; 2015. p. 55-60. doi: 10.11610/posture.08.

6. Dimitrov B, Gatev P. Effects of Acute Transcutaneous Vagal Stimulation on the EEG Power Maps, EEG Sources Distribution and Steadiness of Quiet and Sensory-conflicted Stance. In: Gatev P, Hatzitaki V, editors. Posture, Balance and the Brain. Sofia: Procon; 2015. p. 45-54. doi: 10.11610/posture.07.

7. Sofianidis G, Hatzitaki V. Interpersonal Entrainment in Dancers: Contrasting Timing and Haptic Cues. In: Gatev P, Hatzitaki V, editors. Posture, Balance and the Brain. Sofia: Procon; 2015. p. 36-44. doi: 10.11610/posture.06. 